\title{
Ressignificando um Castelo: um Olhar sobre Ações de Saúde em Rede
}

\author{
Marcela dos Reis Bigatão \\ Mariana Barbosa Pereira ${ }^{2}$ \\ ${ }^{1}$ Universidade de São Paulo, SP, Brasil. \\ ${ }^{2}$ Centro Universitário Herminio Ometto de Araras, SP,Brasil. \\ Rosana Teresa Onocko Campos \\ ${ }^{3}$ Universidade Estadual de Campinas, Campinas, Brasil.
}

Resumo: Este trabalho traz uma breve contextualização sobre a rede de saúde mental de uma grande cidade brasileira pós Reforma Psiquiátrica, e sobre o funcionamento do dispositivo do Apoio Matricial nessa rede, sendo analisada por meio de um caso clínico de uma família composta por várias pessoas diagnosticadas com transtornos graves de saúde mental acompanhada por serviços de saúde dessa rede. O objetivo do trabalho foi analisar, através do relato de um estudo de caso, as intervenções de saúde realizadas em rede, e os investimentos necessários para qualificação do projeto terapêutico das pessoas envolvidas. Realizou-se análise qualitativa das intervenções realizadas por serviços e equipamentos que compõem uma rede de atenção à saúde. As ações ocorreram de forma articulada com os membros da família. Efetivou-se supervisões, reuniões intra eintersetoriais para planejar ações com a família, entendendo a responsabilidade clínica e sanitária das equipes em relação à gravidade do caso. Foram intensificados os atendimentos com os membros da família, a fim de que quaisquer intervenções fossem construídas com essas pessoas, e não para elas. Após a primeira intervenção, a equipe do CAPS continua os atendimentos até o presente momento, assim como a articulação com os outros serviços envolvidos. Concluímos que a intervenção em rede foi um facilitador para uma ação mais integrada, embora não termine aí. O compartilhamento de saberes, experiências e olhares para o caso, construídos a partir da lógica do Apoio Matricial, proporcionou maior empoderamento das equipes para essas discussões e para a continuidade das ações.

Palavras-chave: Apoio Matricial, Saúde Mental, Centro de Saúde, Serviços de Saúde Mental.

\section{Resignifying a Castle: A Look at Health Actions in Network}

\begin{abstract}
This paper provides a brief background on the mental health network of a large Brazilian city after the Psychiatric Reform, and on the functioning of the Matrix Support device on that network, analyzed through a case of a family of several people diagnosed with serious mental health disorders accompanied by health services of that network. The purpose was to analyze, through the report of a case study, health interventions in the network, and investments needed to quantify the therapeutic project. A qualitative analysis of interventions for services and equipment that make up a network of health care for a large municipality was carried out. The actions took place in coordination with the members of the family. Supervisions, intra and intersectoral meetings were conducted to plan actions with the family, understanding the clinical responsibility of the health teams because of the seriousness of the case. Visits with family members were intensified, so that any intervention was built with these people, not for them. After the first intervention, CAPS team continued to offer the assistance, as well as coordination with the other services involved. We concluded that the intervention in the network was a facilitator for a more integrated action, although it is not the ending point. Sharing knowledge, experiences and approaches about the case, built based on the Matrix Support logic, provided greater empowerment of teams for discussions and for the continuing process. The attention to cases such as that one must be a continuous investment in health and intersectoral network.
\end{abstract}

Keywords: Matrix Support, Mental Health, Health Centers, Mental Health Services. 


\title{
Resignificando un Castillo: una Mirada sobre Acciones de Salud en Red
}

\begin{abstract}
Resumen: Este trabajo trae una breve contextualización sobre la red de salud mental de una gran ciudad brasileña post Reforma Psiquiátrica, y sobre el funcionamiento del dispositivo del Apoyo Matricial en esa red, siendo analizada por medio de un caso clínico de una familia compuesta por varias personas diagnosticadas con varios trastornos graves de salud mental acompañada por servicios de salud de esa red. El objetivo del trabajo fue analizar, a través del relato de un estudio de caso, las intervenciones de salud realizadas en red, y las inversiones necesarias para calificar el proyecto terapéutico de las personas involucradas. Se realizó un análisis cualitativo de las intervenciones realizadas por servicios y equipamientos que componen una red de atención a la salud. Las acciones se produjeron de forma articulada con los miembros de la familia. Se realizaron supervisiones, reuniones intra e intersectoriales para planificar acciones con la familia, entendiendo la responsabilidad clínica y sanitaria de los equipos en relación a la gravedad del caso. Se intensificaron los atendimientos a los miembros de la familia, a fin de que cualquier intervención fuera construida con esas personas, y no para ellas. Después de la primera intervención, el equipo del CAPS continúa las atenciones hasta el presente momento, así como la articulación con los otros servicios involucrados. Concluimos que la intervención en red fue un facilitador para una acción más integrada, aunque no termine ahí. El intercambio de saberes, experiencias y miradas para el caso, construidos a partir de la lógica del Apoyo Matricial, proporcionó mayor empoderamiento de los equipos para esas discusiones y para la continuidad de las acciones.
\end{abstract}

Palabras clave: Apoio Matricial, Salud Mental, Centros de Salud, Servicios de Salud Mental.

\section{Introdução}

A mudança do modelo de atenção em saúde mental no Brasil, ocorrida a partir da Reforma Psiquiátrica, produziu novos equipamentos e articulações entre eles, com a priorização do trabalho em rede. Em abril de 2001 foi aprovada a Lei Federal de Saúde Mental, no 10.216, de autoria do deputado Paulo Delgado, depois de 12 anos de tramitação, que regulamenta o processo de Reforma Psiquiátrica no Brasil.

A rede territorial de serviços proposta na Reforma Psiquiátrica inclui centros de atenção psicossocial (CAPS), centros de convivência e cultura assistidos, cooperativas de trabalho protegido (economia solidária), oficinas de geração de renda e residências terapêuticas, descentralizando e territorializando o atendimento em saúde, conforme previsto na Lei Federal que institui o Sistema Único de Saúde (SUS) no Brasil. Esta rede substituiria o modelo arcaico dos manicômios do Brasil.

O primeiro Centro de Atenção Psicossocial Professor Luiz da Rocha Cerqueira, conhecido como CAPS da Rua Itapeva, surgiu em 1986 em São Paulo
(Ribeiro, 2004). A criação desse CAPS e de tantos outros, com outros nomes e lugares, fez parte de um intenso movimento social, inicialmente de trabalhadores de saúde mental, que buscavam a melhoria da assistência no Brasil e denunciavam a situação precária dos hospitais psiquiátricos, que ainda eram o único recurso destinado aos usuários portadores de transtornos mentais (Brasil, 2004).

Nesse contexto, os serviços de saúde mental surgem em vários municípios do país e vão se consolidando como dispositivos eficazes na diminuição de internações e na mudança do modelo assistencial.

Desde a sanção da Lei n ${ }^{\circ} 10.216$ (2001), mudou-se radicalmente o paradigma da atenção ao sofrimento psíquico: fechamento de hospitais psiquiátricos, redução quantitativa dos leitos que continuam funcionando, serviços territoriais diversos espalhados no país todo, atenção à saúde mental na atenção básica (Brasil, 2005). Em 2011, com a instituição da Rede de Atenção Psicossocial (RAPS), ampliam-se os serviços e dispositivos da rede, com objetivo de garantir acesso e qualidade da atenção em saúde mental de forma integrada e territorial (Portaria ${ }^{\circ} 3.088,2011$, Art. $1^{\circ}$, $\S 16)$. (Ver Figura). 


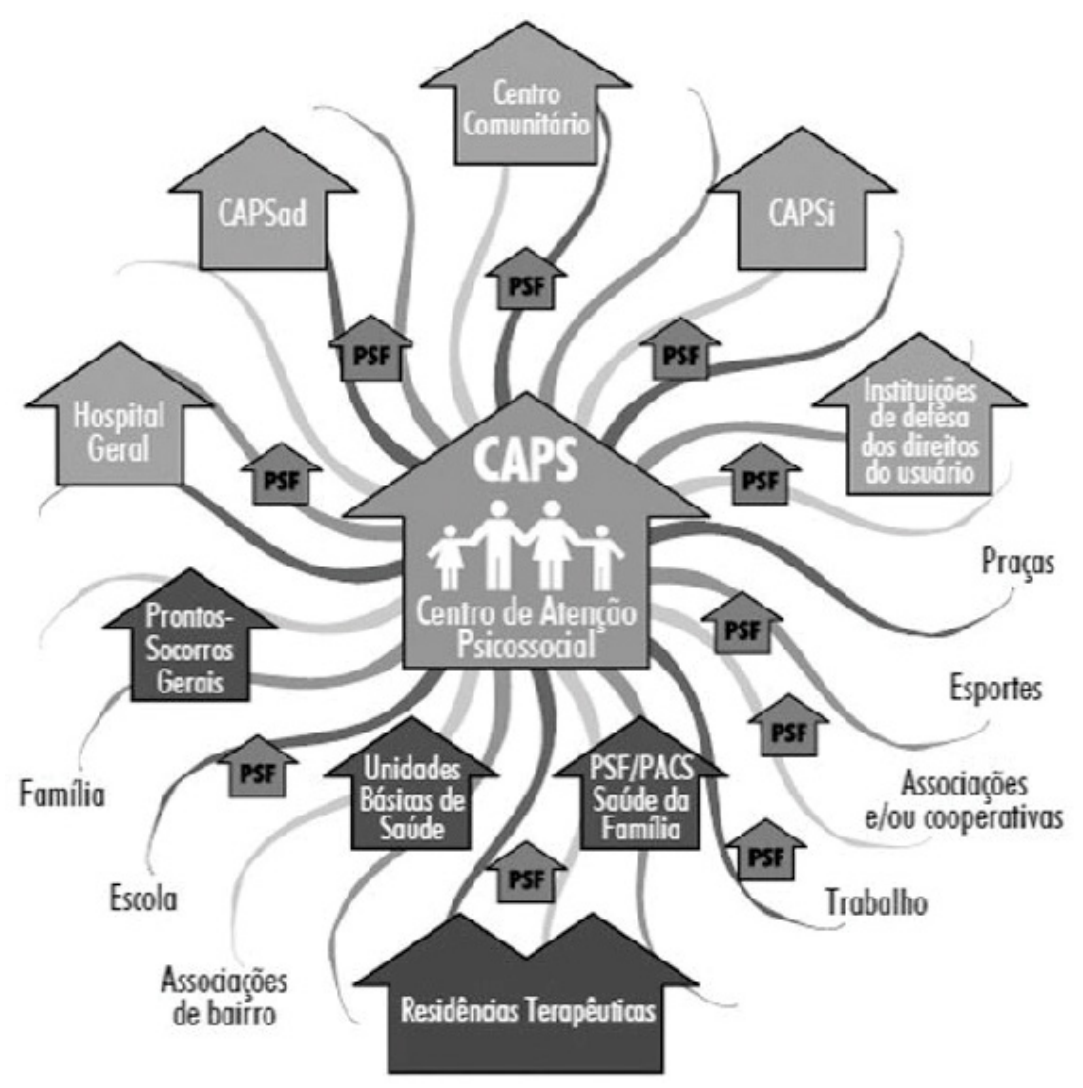

Fonte: Adaptado de Brasil (2004).

\section{Figura 1}

Rede de Atenção Psicossocial.

A RAPS tem por finalidade a criação, "ampliação e articulação de pontos de atenção à saúde para pessoas com sofrimento mental" (Portaria $\mathrm{n}^{\circ} 3.088,2011$, Art. $\left.1^{\circ}, \S 16\right)$. Suas diretrizes convergem com as do SUS e reforça a necessidade do cuidar com autonomia e liberdade, o combate a estigmas e preconceitos, atenção humanizada e centrada nas necessidades das pessoas com construção de projetos terapêuticos singulares, desenvolvimento de estratégias de Redução de Danos e organização dos serviços em rede regionalizada (Portaria ${ }^{\circ} 3.088,2011$ ). Seus objetivos são: "ampliar e promover o acesso à atenção psicossocial e garantir a articulação e integração dos pontos de atenção das redes de saúde no território, qualificando o cuidado por meio do acolhimento, do acompanhamento contínuo e da atenção às urgências”(Portaria $\mathrm{n}^{\circ} 3.088,2011$, Art. $3^{\circ}$, § 33).

A RAPS passa a ser composta pelos seguintes componentes: "Atenção Básica em Saúde (Unidade Básica de Saúde, Consultório na Rua, Atenção Residencial de Caráter Transitório, Centro de Convivên- cia); Atenção Psicossocial Especializada (Centros de Atenção Psicossocial); Atenção de Urgência e Emergência, Atenção Hospitalar; Serviços Residenciais Terapêuticos"(Portaria ${ }^{\circ} 3.088,2011$, Art. $5^{\circ}$, § 44).

No município de Campinas, contexto do caso aqui exposto, a RAPS inclui CAPS, Centros de Convivência, Cooperativas de Trabalho Protegido (economia solidária), Oficinas De Geração De Renda e Serviços Residenciais Terapêuticos, descentralizando e territorializando o atendimento em saúde, conforme previsto na Lei Federal que institui o SUS no Brasil (Prefeitura de Campinas, 2017; Lei n 8.080, 1990).

O objetivo dos CAPS, um dos equipamentos centrais da RAPS, "é oferecer atendimento à população de sua área de abrangência, realizando o acompanhamento clínico e a reinserção social dos usuários pelo acesso ao trabalho, lazer, exercício dos direitos civis e fortalecimento dos laços familiares e comunitários"(Brasil, 2004, p. 13). Para atingir esse objetivo, o CAPS deve funcionar em rede e no território. 
A Rede Básica de Saúde é considerada "o lugar privilegiado de construção de uma nova lógica de atendimento e de relação com os transtornos mentais"(Brasil, 2004, p. 25). A atenção básica em saúde se constitui por:

Centros ou unidades de saúde locais e/ou regionais, pelo Programa de Saúde da Família e de Agentes Comunitários de Saúde, que atuam na comunidade de sua área de abrangência. Esses profissionais e equipes são pessoas que estão próximas e que possuem a responsabilidade pela atenção à saúde da população daquele território (Brasil, 2004, p. 25).

Os CAPS devem buscar uma integração permanente com as equipes da rede básica de saúde em seu território, pois têm um papel fundamental no acompanhamento, na capacitação e no apoio para o trabalho dessas equipes com as pessoas com transtornos mentais.

Um recurso estratégico para o compartilhamento dos casos entre serviços da rede é o Apoio Matricial, que opera de modo diferente da lógica de encaminhamento ou da referência e contra-referência no sentido estrito, porque supõe a corresponsabilização (Brasil, 2004).

O Apoio Matricial, ou Matriciamento, representa uma nova forma de gestão em saúde que busca romper com os modelos das organizações substancialmente tayloristas, com vistas ao fortalecimento de métodos organizativos pautados na cogestão (Campos, 1998).

Quando o território for constituído por uma grande população de abrangência, é importante que o CAPS discuta com o gestor local a possibilidade de acrescentar a seu corpo funcional uma ou mais equipes de saúde mental, destinadas a realizar essas atividades de apoio à rede básica. Essas atividades não devem assumir características de uma "especialização", devem estar integradas completamente ao funcionamento geral do CAPS. As atuais diretrizes orientam que, onde houver cobertura do Programa de Saúde da Família, deverá haver uma equipe de apoio matricial em saúde mental para no mínimo seis e no máximo nove equipes de PSF (Brasil, 2004, p. 25).
Contudo, ainda é um desafio a criação de espaços coletivos, nos quais as equipes de saúde compartilhem a elaboração de planos gerenciais e projetos terapêuticos, principalmente devido à tendência hegemônica de fragmentação do trabalho, obstáculo estrutural à clínica ampliada e ao trabalho interdisciplinar (Campos, 1998). Para Campos (2003), “O Apoio Matricial é uma ferramenta para agenciar a indispensável instrumentalização das equipes na ampliação da clínica" (p. 9).

Segundo Figueiredo e Onocko-Campos (2009): A experimentação deste arranjo foi inaugurada no município de Campinas (SP) a partir de 2001, com a implantação do Programa Paidéia Saúde da Família, uma adaptação do Programa de Saúde da Família do Ministério da Saúde, ajustado ao contexto sanitário local (p. 130).

A estratégia do Apoio Matricial visa um suporte especializado a equipes e profissionais de saúde, como metodologia de trabalho complementar às já estabelecidas no setor da saúde para relação entre serviços, como mecanismos de referência e contrarreferência, protocolos e centros de regulação. Esse suporte pode ser tanto assistencial quanto técnico-pedagógico, a depender das demandas específicas dos casos acompanhados e das equipes. É essencial, invariavelmente, que sejam estabelecidos critérios claros para corresponsabilização pelo trabalho entre a equipe de referência e os apoiadores matriciais (Campos, \& Domitti, 2007).

A ideia é que, a partir de um suporte técnico especializado, a equipe interdisciplinar possa ampliar e qualificar seu campo de atuação e suas ações locais. No caso específico da saúde mental, uma consequência da qualificação das ações é poder identificar e distinguir situações individuais e sociais que não demandam necessariamente recursos da atenção especializada em saúde mental, daquelas situações que possuem gravidade ou risco que indicam necessidade de encaminhamento a cuidados especializados. Dessa forma, busca-se evitar práticas no sentido da "psiquiatrização" e "medicalização" do sofrimento. Ao mesmo tempo, trabalha-se para garantir o acesso de acordo com o princípio da equidade, avaliando as vulnerabilidades e potencialidades de cada usuário (Figueiredo, \& Onocko-Campos, 2009).

A partir dos conceitos brevemente expostos, acerca de possibilidades de funcionamento da rede de atenção em saúde mental, apresentaremos o caso 
de uma família, cujo contexto é compreendido como socialmente vulnerável, de adoecimento psíquico e dificuldades relacionais no núcleo familiar. A família foi acompanhada por alguns equipamentos de saúde e assistência social (CAPS, Atenção Básica, Vigilância Sanitária, Centro de Referência de Assistência Social - CRAS), incluindo-se o recurso do Apoio Matricial, visando um cuidado integral e singularizado.

\section{Método}

Análise qualitativa das intervenções realizadas por serviços e equipamentos que compõem uma rede de atenção à saúde (CAPS, Unidade Básica de Saúde - UBS, Assistência Social, Vigilância Sanitária) de um município de grande porte a partir do método clínico-qualitativo (Turato, 2005). As ações ocorreram de forma articulada com os membros da família, compreendidos como os principais atores no processo.

A metodologia utilizada relaciona-se especificamente com os settings das vivências em saúde. Trata-se de analisar os significados - de natureza psicológica e complementarmente sociocultural - trazidos por indivíduos (pacientes ou outras pessoas que lidam com problemas da saúde, tais como familiares, profissionais de saúde e sujeitos da comunidade), acerca dos múltiplos fenômenos pertinentes ao campo dos problemas da saúde-doença (Turato, 2005).

Através do relato de estudo de caso, objetiva-se analisar as intervenções de saúde realizadas em rede, e os investimentos necessários para qualificação do projeto terapêutico das pessoas envolvidas.

\section{Caso clínico}

Segundo Serapioni (2005) "A família forma a unidade básica da organização social na nossa sociedade. Para alguém ela representa o pré-requisito de um sistema social estável" (p. 245).

A família que constitui o caso aqui apresentado é composta por HV (81 anos) e JV (já falecido), que eram casados e tiveram quatro filhos: DV (44 anos); ARV (40 anos); EV (38 anos); e RV (que faleceu aos 13 anos devido a uma infecção na garganta, segundo HV). Todos os membros da família residem juntos.

Os três filhos apresentam um comprometimento cognitivo, porém em diferentes níveis. As duas filhas estão inseridas e são regularmente acompanhadas por um serviço de saúde mental (CAPS); o filho trabalha em uma empresa em outra cidade, e não é acom- panhado por nenhum serviço especializado em saúde mental. Atualmente, $\mathrm{HV}$ recebe a pensão do marido e é quem sustenta financeiramente a casa (embora $\mathrm{EV}$ trabalhe, sua renda não consegue contribuir substancialmente para as despesas da casa).

Em 2006, após queixas de vizinhos (que reclamavam especialmente sobre o mau cheiro e o acúmulo de objetos e animais na casa) e avaliação da UBS, houve uma intervenção pela Vigilância Sanitária, na residência. A casa se encontrava com lixo e objetos acumulados (roupas, sapatos, baldes, sacolas etc), insetos, roedores e outros animais que provocam doenças, como leptospirose, febre tifoide, pneumonia, verminoses, tétano, dermatite de contato etc. Após essa intervenção, observou-se que JV (que havia sofrido um acidente vascular cerebral (AVC) e estava acamado) e HV não apresentavam saúde adequada e foram encaminhados ao Pronto Socorro de um hospital da cidade.

Após melhora do quadro clínico, foi realizado encaminhamento de JV para o Centro de Referência do Idoso. Nesse período, ele solicita para ser abrigado por tempo determinado, até sua recuperação. Pouco tempo depois do abrigamento, JV vai a óbito, o que leva HV a responsabilizar a UBS e o CAPS pelo ocorrido. Inicia-se, então, uma relação difícil entre a família e os serviços de saúde, baseada na persecutoriedade, desconfiança e sentimento de invasão, em especial por parte de HV.

Em abril de 2013, durante visita domiciliar à família, a equipe do CAPS observa que HV se encontrava extremamente debilitada clinicamente, avaliando a necessidade de uma intervenção em caráter de urgência. Naquele momento, foram feitas tentativas de contato com outros familiares, de modo a acionar uma rede de apoio, porém HV refere não ter mais familiares próximos.

O recurso da visita domiciliar é bastante utilizado, tanto pelas equipes dos CAPS, quanto das UBS, e com diversos fins. A visita domiciliar compõe os recursos para se aproximar da realidade dos usuários e do território, portanto, faz-se necessária a avaliação do seu papel e a discussão dos conceitos de acesso e cuidado a serem realizados pela equipe que trabalha nesta proposta (Abrahão, \& Lagrange, 2007).

De acordo com Coelho e Savassi (2011), a visita pode ser realizada de duas formas. Uma primeira seria denominada visita Fim, e teria objetivos direcionados a acompanhamentos já estruturados; em 
geral, à atenção domiciliar terapêutica e visita a pacientes acamados. O tipo de visita denominada pelos autores de Meio teria como objetivos a prevenção e promoção de saúde, com ações voltadas para educação em saúde, e mesmo busca ativa de demandas reprimidas.

Seja qual for seu objetivo, corroborando com Drulla, Alexandre, Rubel e Mazza (2009), acreditamos que a compreensão da visita domiciliar desenvolvida pela equipe de Saúde da Família deve possibilitar uma reflexão sobre essa prática, identificando e explicitando tanto seu potencial para os cuidados com as famílias, quanto as dificuldades de realização desse tipo de ação como construção coletiva da equipe.

A partir dessas reflexões, podemos pensar que se trata de um desafio para as equipes de saúde, e é necessário que estejam dispostas a trabalhar dialogicamente entre si, e com os usuários. Nesse sentido, a visita domiciliar pode proporcionar "um cuidado mais humanizado, permitindo a construção de vínculo. Para tanto, é preciso saber ouvir, para que se possa estabelecer uma ligação de confiança entre o profissional e, especialmente, o usuário, pois esta prática é desenvolvida no espaço domiciliar familiar" (Drulla et al., 2009, p. 673).

As primeiras discussões em equipe sobre o caso da família em questão giraram em torno dos direitos do idoso, entendendo que $\mathrm{HV}$ teria a prerrogativa legal de melhores condições de cuidados e, caso tivesse algum familiar (não sendo os filhos), este deveria ser corresponsabilizado, caso contrário, seria atribuído ao poder público por meio da Lei $\mathrm{n}^{\circ} 10.741$ de 2003, que trata dos direitos do idoso.

De imediato, discutiu-se o caso com o Centro de Saúde (CS) para auxiliar nos primeiros cuidados básicos. Contudo, houve uma resistência inicial da equipe do Programa de Saúde da Família (PSF) pelo fato da idosa sempre culpabilizar o serviço por tudo de ruim que lhe aconteceu. Apesar dessa resistência inicial, a equipe do CS foi ao domicílio e confirmou a avaliação de que seria necessária uma internação hospitalar, devido a uma suspeita de pneumonia, confirmada posteriormente no hospital.

Vale lembrar que resistência e transferência são peças fundamentais no tratamento psicológico. Em virtude disso, afirma-se que o grande e árduo trabalho da análise é o manejo da transferência, sendo denominada de "motor da resistência", pois por si só é uma resistência e as questões inconscientes favo- recem seu aparecimento (Freud, 1980). Ao longo do acompanhamento da família, foi necessário, como em todo processo terapêutico, reconhecer e lidar com esses fatores.

Após a hospitalização de HV devido à pneumonia bacteriana, realizou-se visita no hospital e a assistente social da instituição identificou duas familiares da usuária, que teriam ido a algumas visitas. As equipes de saúde procuraram, então, os familiares, propondo uma conversa sobre as dificuldades enfrentadas pela família, e naquele momento mostraram-se disponíveis para isso. Foram expostas as principais dificuldades da família, e o fato de que, após o adoecimento de $\mathrm{HV}$, seria necessário pensar em uma intervenção na casa, devido aos problemas causados pelo acúmulo de objetos, sujeira, animais etc.

Podemos dizer que, a partir da visita, realizada pela equipe do CAPS, a HV no hospital, iniciou-se a construção de um vínculo com a usuária, e o que viria a ser posteriormente, com a família. De acordo com Pichon-Rivière (1982), o vínculo "é um tipo particular de relação com o objeto; a relação com o objeto é constituída por uma estrutura que funciona de uma determinada maneira" (p. 17).

Primeiramente, discutiu-se em Matriciamento com a equipe PSF e em conjunto se decidiu por marcar uma reunião com outros serviços da região para pensar nas estratégias de ação.

O Matriciamento entre CS e CAPS acontece mensalmente no próprio CS, onde nessa reunião o PSF prioriza os casos da saúde mental e o CAPS, os casos com alguma questão clínica ou sociofamiliar, porém em todas as reuniões há um compartilhamento dos casos que são de difícil manejo para as equipes. Nessas reuniões também ocorrem a transferência de cuidados de ambas as partes, pois a situações em que o sujeito necessite em algum momento do CAPS e posteriormente se restabelece e fica no cuidado do CS e, ao contrário também ocorre.

A relação entre CAPS e CS nem sempre é fácil, há discussões acaloradas e relações estremecidas, principalmente da categoria médica, que nem sempre se dispõe a participar dos matriciamentos. Quando os agentes de saúde participam das reuniões sempre acrescentam algo muito enriquecedor para a discussão do caso. Quando há disponibilidade de ambas as equipes consegue-se traçar um Projeto Terapêutico Singular (PTS) mais compartilhado para o sujeito/ família. Nesse caso específico 
isso aconteceu, havia disponibilidade de cuidar dessa família. Assim dentre contradições e corroborações, foi traçado um PTS.

Para a próxima reunião convidou-se a vigilância sanitária, o centro de saúde, o centro de convivência e o CAPS, para pensar juntos como fazer essa intervenção de forma diferente da anterior, nessa reunião também estava uma supervisora institucional. Procurava-se estabelecer diálogo e pontes entre concepções de mundo distintas. Nesse sentido, reconhece-se que a "presença de um terceiro (apoiador, profissional clínico, mestre etc.), que se valha do recurso da cogestão nas relações humanas, facilita o processo de mediação de conflitos e de co-governo da vida em sociedade" (Campos, Cunha, \& Figueiredo, 2013, p. 7).

O CS iniciou contando como havia sido difícil a intervenção anterior e explicando a resistência de HV frente à equipe de saúde. Levantaram-se conjuntamente os pontos positivos e negativos da intervenção de 2006, para refletir sobre como se desejava que fosse a próxima.

A equipe do CAPS tinha a preocupação de ser o menos invasivo possível e de que a família pudesse participar ativamente desse processo de intervenção.

A utilização do conceito de Projeto Terapêutico Singular pressupõe a participação do sujeito na formulação e andamento do tratamento, afinal não se muda algo para alguém, senão com esse alguém. É esse alguém que vai acompanhar em seus modos de andar na vida. Então é preciso refletir junto com o próprio sujeito: Para onde se quer ir? Que mudanças (trajetos) se querem produzir? Como será feito isso? (Figueiredo, \& Onocko-Campos, 2009).

Cunha (2009) define quatro movimentos na construção de um PTS:

Definição de hipóteses diagnósticas ou problemas: deverá partir de algum consenso sobre os problemas relevantes tanto para a equipe, quanto para o(s) usuário(s); 2. Definição de metas: propostas de curto, médio e longo prazo que serão negociadas com o(s) usuário(s) e os membros da equipe envolvidos; 3 . Divisão de responsabilidades: definir as tarefas de cada um com clareza. Os membros da equipe que possuem vínculo mais estreito com o(s) usuário(s) se tornam referência na coordenação do PTS. 4. Reavaliação: discutir a evolução e fazer as correções dos rumos tomados (pp. 23-25).
A vigilância sanitária colocou a preocupação de não estar sozinha na intervenção sobre os insetos e roedores, propondo estratégias para exterminá-los antes da intervenção. Entendeu-se que para uma intervenção desse tipo era necessário contar com ações integrais.

O conceito de integralidade remete, portanto, obrigatoriamente, ao deintegração de serviços por meio de redes assistenciais, reconhecendo a interdependência dos atores e organizações, em face da constatação de que nenhuma delas dispõe da totalidade dos recursos e competências necessários para a solução dos problemas de saúde de uma população em seus diversos ciclos de vida. Torna-se, portanto, indispensável desenvolver mecanismos de cooperação e coordenação próprios de uma gestão eficiente e responsável dos recursos coletivos, a qual responda às necessidades de saúde individuais em âmbitos local e regional (Hartz, \& Contandriopoulos, 2004, pp. 331- 332).

Foi agendada uma data para um mês e meio após a reunião, período durante o qual o CAPS intensificaria os atendimentos, incluiria a família nas decisões e, assim que se avaliasse ser possível a intervenção na casa, seria realizado contato telefônico com os serviços e se definiria a data.

O CAPS, representado por uma terapeuta ocupacional e uma psicóloga, intensificou os atendimentos domiciliares, porém ainda não haviam acessado a casa, desconhecendo o que encontrariam lá. Iniciou-se a conversa com HV e ARV sobre a saúde da família, a preocupação de que a pneumonia fosse decorrente do acúmulo de coisas na casa e elas se mostraram atentas.

Após algumas visitas/atendimentos conseguiram ser convidadas a entrar na casa. Havia muitas coisas. Identificaram que cada membro da família era responsável por acumular objetos diferentes. HV acumulava produtos de limpeza, EV armazenava reciclagem e passarinhos e ARV amontoava objetos de diversas ordens como roupas, sapatos, bolsas, pulseiras, tudo que encontrava na rua. Somente DV não acumulava.

Assim, foi priorizado o atendimento da ARV, e começou-se junto com ela a separar alguns sapatos. Como se estivesse vivendo a história da Cinderela, ela ia experimentando os sapatos e as profissionais intervinham no sentido de questionar o que poderia ser por ela usado e, eram separados. Naquele aten- 
dimento, foram separados três sacos de sapatos, o que foi muito sofrido para ARV, que chorou muito e dizia ser aquele o seu castelo. Segundo Fazio citado em Almeida e Pontes (2014), "o entulho, na visão do acumulador, não é lixo, é a riqueza de sua vida” (p. 3). HV, então, refere um desejo, de que o quarto de ARV pudesse ser preenchido com outros objetos, como uma penteadeira, e isso inspirou os cuidados seguintes. Assim, o quarto de acúmulo de coisas até o teto poderia ser preenchido com móveis de quarto (cama, armário e penteadeira). Produziu-se, desta forma, a participação do usuário na ação como protagonista da sua história. Esta foi uma cena escolhida para relatar um dos atendimentos na casa.

No dia da intervenção, estavam lá todos das equipes de saúde, vigilância sanitária, e família. Iniciou-se a retirada dos objetos, havendo preparação e com o frequente consentimento dos membros da família para que se pudesse descartar esse ou aquele objeto. Segundo Dejuan et al. (1993), sem seu acordo, desencadeia-se nos acumuladores a persecutoriedade, a agitação clástica, o mutismo. Tratava-se de promover uma atmosfera neutra para a experiência, na qual o acordo mútuo desqualificava as noções de consentimento ou de submissão. Quando a casa começou a ficar vazia, ou melhor, com menos coisas, a ARV iniciou um choro intenso e ficou muito brava, sendo acolhido seu sofrimento, enquanto a DV admirava seu quarto ter chão. Após três caminhões carregados de objetos saírem, a família foi levada para o CAPS para se alimentar, descansar e serem acolhidos.

A acumulação compulsiva é um sintoma, que reflete profunda desorganização psíquica. Embora os sintomas de acumulação compulsiva sejam inicialmente relatados na meia-idade, as causas são consideradas na infância ou na adolescência. Nenhum indivíduo relatou o início da acumulação compulsiva tardiamente. A acumulação compulsiva torna-se mais severa e aumenta a cada década de vida (Ayers, Saxena, Golshan, \& Wetherell, 2010).

Segundo Lima (2011), existe:

Uma linha tênue entre o estilo acumulador compulsivo e o estilo colecionador, esse último [...] tende a organização dos objetos, calcula o espaço e valores. Já os acumuladores compulsivos são incapazes de organizar o seu espaço de convivência; na verdade, eles perderam o autocontrole para adquirir ou não se desfazer das coisas, que, no fundo, são investimentos simbólicos alheios a sua consciência (p. 208).

Assim, "Prevalece o medo de tomar a decisão errada e perder os referenciais, os itens materiais que marcaram a experiência de viver [...] para estes pacientes, a escolha segura é postergar a decisão, ou seja, amontoar" (Modenese, 2011, p. 12).

\section{Resultados e Discussão}

Logo após a intervenção, HV mostrou-se resistente ao contato, acusando às equipes pela destruição do castelo, porém persistiu-se com as visitas domiciliares, até que ela aceitou receber novamente às profissionais. Aquela intervenção foi ressignificada e começou-se a planejar o quarto. Angariou-se camas e colchões e um artista plástico do centro de convivência pintaria o quarto com as cores escolhidas por ARV e DV. Entretanto, as tintas demoram a chegar e isso desestimulou ARV que iniciou novamente a recolher objetos nas ruas e acumular em sua casa.

A situação de saúde de forte predomínio relativo das condições crônicas não pode ser respondida, com eficiência, efetividade e qualidade, por sistemas de saúde voltados, prioritariamente, para as condições agudas e para as agudizações de condições crônicas, e organizados de forma fragmentada (Mendes, 2010, p. 2298).

Como o PTS não é estático, rediscutiu-se entre as equipes em nova reunião sendo proposto que ARV fosse também acompanhada no Centro de Convivência perto de sua casa, local do qual ela fazia bom uso, pois o CAPS ela frequentava irregularmente. No Centro de Convivência, ARV começou a trocar objetos, como, por exemplo, roupas e pulseiras ao invés de levá-los para casa.

DV continuou a frequência diária ao CAPS para manter seus cuidados e o CAPS prosseguiu realizando as visitas domiciliares e sempre discutindo com o CS sobre os cuidados com a família. Também se fez contato com a equipe da assistência social para verificar possibilidades de parcerias, porém esta não identificou demanda junto a família.

Nesse processo nota-se a necessidade de sempre olhar o todo (família) e ao mesmo tempo, vislumbrar o individual (ARV, HV, DV, EV). Segundo Morin (2000), 
assim como cada ponto singular de um holograma contém a totalidade da informação do que representa, cada célula singular, cada indivíduo singular contém de maneira "hologrâmica" o todo do qual faz parte e que, ao mesmo tempo, faz parte dele (p. 37-38).

Isso é um conhecimento complexus, que para Morin (2000) "significa o que foi tecido junto; complexidade é a união entre a unidade e a multiplicidade" (p. 38).

Sabe-se que a implantação do SUS e, portanto, as ações articuladas em rede, é um processo inacabado e os desafios não cessam (Campos, \& Domitti, 2007). Entre outros: as desigualdades sociais continuam sendo altas; as articulações público-privado afetam de forma negativa as condições de saúde, a equidade e o acesso aos serviços. Assim, a porcentagem destinada ao setor de saúde no orçamento federal é baixa; continua sendo necessário melhorar a qualidade da atenção, as condições de trabalho dos trabalhadores e a gestão do sistema. A fragmentação das políticas públicas contribui para o acirramento dessas dificuldades (World Health Organization, 2008).

No caso da saúde mental, o respaldo legal para a desinstitucionalização, a partir da Lei ${ }^{\circ} 10.216$ de 2001, possibilitou que a luta pela garantia dos direitos de pessoas com transtornos mentais graves fosse legitimada e contemplada nas políticas públicas. Ainda assim, é necessário destacar que os desafios associados à necessidade de maior integração das políticas públicas em saúde tem grande impacto na atenção a casos como o que foi apresentado, em que a articulação de serviços, arranjos e dispositivos é crucial para sua condução (Onocko-Campos, 2012).

Considerando tais dificuldades, a condução do caso apresentado demonstrou a possibilidade de cuidado humanizado em contextos de grande vulnerabilidade social e psíquica, quando de fato se concretiza o trabalho articulado em rede, e quando são consideradas as realidades subjetivas daqueles a quem se dirigem as intervenções em saúde.

A construção de vínculo das equipes com a família foi ferramenta central para o manejo de um caso com essa complexidade e diversidade, considerando a existência de vários indivíduos na mesma família, assim como de diferentes equipes e serviços envolvidos.

\section{Conclusão}

Compreendemos, a partir da condução deste caso, que a atenção a famílias que apresentam esse tipo de desorganização psíquica e espacial deve ser um investimento contínuo da rede de saúde e assistência. Quando realizada conjuntamente, em rede e com ações e discussões compartilhadas, esse tipo de atenção produz efeitos positivos.

O trabalho com a família reforçou a avaliação da importância dos CAPS III, não apenas no sentido da sua capacidade de acolher a crise e evitar internações em hospitais psiquiátricos, mas de sua inserção efetiva no território. O Apoio Matricial fortaleceu teoricamente a discussão do caso, e também empoderou a articulação dessa rede de saúde.

Nesse sentido, enfatizamos a necessidade de fortalecimento de ações em saúde mental no âmbito da atenção primária e articuladas em rede, o que torna mais viável o acesso aos serviços, além de evitar custos indiretos associados à procura de cuidados especializados em locais distantes. Cuidados de saúde mental prestados neste nível podem minimizar o estigma e a discriminação, assim como o risco de violações de direitos humanos que ocorrem em hospitais psiquiátricos. Há maior possibilidade de singularização da relação entre usuário e equipe, de descentralização do papel do profissional médico, e da participação e protagonismo de todos os cidadãos. Por outro lado, sabe-se que a integração de serviços de saúde mental nos cuidados primários gera bons resultados de saúde a custos razoáveis (Lancetti, 2001; Word Health Organization, 2013; World Health Organization, 2008).

São ainda incipientes os investimentos na atenção primária com foco em ações que visem aprimorar o potencial de resolução de problemas de saúde mental. Tais ações incluem capacitações e programas de educação permanente, organização em equipes de referência para um conjunto de casos, garantia de espaços coletivos na equipe de discussão de casos e elaboração de projetos terapêuticos, supervisões clínico-institucionais, apoio matricial (Campos, \& Guerrero, 2008). Casos como o relatado aqui, argumentam a favor da necessidade de continuidade desses investimentos.

Serviços e profissionais da rede se conheceram, compartilharam dificuldades do trabalho em um sistema ainda com pouco investimento em termos de recursos financeiros e humanos, e também identificaram o potencial dos recursos individuais e das equipes. A valorização das experiências positivas no âmbito dos serviços públicos de saúde é condição, tanto para o fortalecimento das próprias equipes, quanto para a reivindicação de recursos e melhorias necessários. 


\section{Referências}

Abrahão, A. L., \& Lagrange, V. (2007). A visita domiciliar como uma estratégia da assistência no domicílio. In: M. V. G. C. Morosini, \& A. D. Corbo (Orgs.), Modelos de atenção e a saúde da família (pp. 151-171). Rio de Janeiro, RJ: Escola Politécnica de Saúde Joaquim Venâncio.

Almeida, G. L., \& Pontes, H. H. S. (2014). Disposofobia: A função existencial de acumular. Caderno de Ciências Biológicas e da Saúde, (4), 1-13.

Ayers, C. R., Saxena, S., Golshan, S., \&Wetherell, J. L. (2010). Age at onset and clinical features of late life compulsive hoarding. International Journal of Geriatric Psychiatry, 25(2), 142-149. https:// doi.org/10.1002/gps.2310

Brasil. Ministério da Saúde. (2004). Saúde mental no SUS: Os centros de atenção psicossocial. Brasília, DF: o autor. Recuperado de http://www.ccs.saude.gov.br/saude_mental/pdf/sm_sus.pdf

Brasil. Ministério da Saúde. (2005). Reforma psiquiátrica e política de saúde mental no Brasil. Trabalho apresentado na Conferência Regional da Reforma dos Serviços de Saúde Mental: 15 anos depois de Caracas, Brasília, DF. https:// doi.org/10.1590/S0101-73302003000400015

Campos, G. W. D. S. (1998). O anti-Taylor: Sobre a invenção de um método para co-governar instituições de saúde produzindo liberdade e compromisso. Cadernos de Saúde Pública, 14(4), 863-870. https://doi.org/10.1590/ S0102-311X1998000400029

Campos, G. W. D. S. (2003). A clínica do sujeito: Por uma clínica reformulada e ampliada. In: G. W. D. S. Campos (Org.), Saúde Paidéia (p. 51-67). São Paulo, SP: Hucitec.

Campos, G. W. D. S., Cunha, G. T., \& Figueiredo, M. D. (2013). Práxis e formação paideia: Apoio e cogestão em saúde. São Paulo, SP: Hucitec.

Campos, G.W.S., \& Domitti, A. C. (2007). Apoio matricial e equipe de referência: Uma metodologia para gestão do trabalho interdisciplinar em saúde. Cadernos de Saúde Pública, 23(2), 399-407. http://doi.org/10.1590/S0102$311 X 2007000200016$

Campos, G. W. D. S., \& Guerrero, A. V. P. (2008). Manual de práticas em atenção básica: Saúde ampliada e compartilhada. São Paulo, SP: Hucitec.

Coelho, F. L. G., \& Savassi, L. C. M. (2011). Aplicação de escala de risco familiar como instrumento de priorização das visitas domiciliares. Revista Brasileira de Medicina de Família e Comunidade, 1(2), 19-26. https://doi. org/10.5712/rbmfcl(2)104

Cunha, G. T. (2009). Grupos balint paideia: Uma contribuição para a co-gestão e a clinica ampliada na atenção basica (Tese de Doutorado). Universidade Estadual de Campinas, Campinas, SP.

Dejuan, P., Drogoul, F., Giraudon, L., Leclerc, S., Leroux, A., Moreau, S. et al. (1993). Arrumação e limpeza, espaço, psicose. Revista Institutions L'aliénation, (12).

Drulla, A. G., Alexandre, A. M. C., Rubel, F. I., \& Mazza, V. A. (2009). A visita domiciliar como ferramenta ao cuidado familiar. Cogitare Enfermagem, 14(4), 667-674. http://dx.doi.org/10.5380/ce.v14i4.16380

Figueiredo, M. D., \& Onocko-Campos, R. (2009). Saúde Mental na atenção básica à saúde de Campinas, SP: Uma rede ou um emaranhado? Ciência \& Saúde Coletiva, 14(1), 129-138. https://doi.org/10.1590/S141381232009000100018

Freud, S. (1980). A dinâmica da transferência. In: J. Salomão (Org.), Edição standard brasileira de obras completas de Sigmund Freud (pp. 131-143). Rio de Janeiro, RJ: Imago.

Hartz, Z. M. A., \& Contandriopoulos, A. -P. (2004). Integralidade da atenção e integração de serviços de saúde: Desafios para avaliar a implantação de um “sistema sem muros”. Cadernos de Saúde Pública, 20(supl. 2), 331 -336. https:// doi.org/10.1590/S0102-311X2004000800026

Lancetti, A. (2001). Saúde loucura, Vol. 7: Saúde mental e saúde da família. São Paulo, SP: Hucitec.

Lei $N^{\circ}$ 8.080, de 19 de setembro de 1990. Dispõe sobre as condições para a promoção, proteção e recuperação da saúde, a organização e o funcionamento dos serviços correspondentes e dá outras providências. Diário Oficial da União, 19 set. 1990. Recuperado de http://www.planalto.gov.br/ccivil_03/leis/L8080.htm 
Lei $N^{o} 10.216$, de 6 de abril de 2001. Dispõe sobre a proteção e os direitos das pessoas portadoras de transtornos mentais e redireciona o modelo assistencial em saúde mental. Diário Oficial da União, 7 abr. 2001. Recuperado de http://www.planalto.gov.br/ccivil_03/leis/leis_2001/110216.htm

Lei $\mathrm{N}^{\circ} 10.741$, de 1 de outubro de 2003. Dispõe sobre o estatuto do idoso e dá outras providências. Diário Oficial da União, 2 out. 2003. Recuperado de http://www.planalto.gov.br/ccivil_03/leis/2003/L10.741.htm

Lima, R. (2011). Acumuladores compulsivos: Uma nova patologia psíquica. Revista Espaço acadêmico, 11(126), 208-215.

Mendes, E. V. (2010). As redes de atenção à saúde. Ciência \& Saúde Coletiva, 15(5), 2297-2305. https://doi. org/10.1590/S1413-81232010000500005

Modenese, F. (2011). Quando colecionar se torna obsessão. Jornal da Faculdade de Medicina de Botucatu, 3(31), 1-12.

Morin, E. (2000). Os setes saberes necessários à educação do futuro (2a ed., C. E. F. Silva, \& J. Sawaya, trads.). Brasília, DF: Unesco.

Onocko-Campos, R. (2012). Humano demasiado humano: Uma abordagem do mal-estar na instituição hospitalar. In: R. Onocko-Campos (Org.), Psicoanálise e saúde coletiva: Interfaces (pp. 57-76). São Paulo, SP: Hucitec.

Pichon-Rivière, E. (1982). Teoria do vínculo. São Paulo, SP: Teoria do vínculo.

Portaria $\mathrm{N}^{\circ} 3.088$, de 23 de dezembro de 2011. Institui a rede de atenção psicossocial para pessoas com sofrimento ou transtorno mental e com necessidades decorrentes do uso de crack, álcool e outras drogas, no âmbito do sistema único de saúde (SUS). Diário Oficial da União, 24 de dez. 2011. Recuperado de http://bvsms.saude.gov.br/ bvs/saudelegis/gm/2011/prt3088_23_12_2011_rep.html

Prefeitura de Campinas. Secretária Municipal de Saúde. (2017). Estrutura do SUS Campinas. Campinas, SP: o autor. Recuperado de http://www.saude.campinas.sp.gov.br/saude/

Ribeiro, S. L. (2004). A criação do Centro de Atenção Psicossocial Espaço Vivo. Psicologia ciências e profissões, 24(3), 92-99. https://doi.org/10.1590/S1414-98932004000300012

Serapioni, M. (2005). O papel da família e das redes primárias na reestruturação das políticas sociais. Ciência \& Saúde Coletiva, 10(supl.), 243-245. https://doi.org/10.1590/S1413-81232005000500025

Turato, E. R. (2005). Métodos qualitativos e quantitativos na área da saúde: Definições, diferenças e seus objetos de pesquisa. Revista de Saude Publica, 39(3), 507-514. https:// doi.org/10.1590/S0034-89102005000300025

Word Health Organization - WHO. (2013). Mental health action plan 2013-2020. Geneva: o autor. Recuperado de http://apps.who.int/iris/bitstream/10665/89966/1/9789241506021_eng.pdf?ua=1

World Health Organization -WHO. (2008). Integrating mental health and primary care: A global perspective. Singapore: $\mathrm{o}$ autor.

Abrahão, A. L., \& Lagrange, V. (2007). A visita domiciliar como uma estratégia da assistência no domicílio. In M. V. G. C. Morosini \& A. D. Corbo (Orgs.), Modelos de atenção e a saúde da família (p. 151-171). Rio de Janeiro, RJ: Escola Politécnica de Saúde Joaquim Venâncio; FIOCRUZ. Disponível em: http://pesquisa.bvs.br/aps/resource/es/sus-18384

\section{Marcela dos Reis Bigatão}

Terapeuta Ocupacional. Doutora em Ciências pela Faculdade de Medicina de Ribeirão Preto, Universidade de São Paulo, Ribeirão Preto - SP. Brasil.

E-mail: marcelarb.to@gmail.com

http://orcid.org/0000-0003-2162-2397

\section{Mariana Barbosa Pereira}

Coordenadora da Saúde Mental do município de Itatiba. Itatiba - SP. Brasil. Docente do curso de Psicologia do Centro Universitário Herminio Ometto de Araras (Uniararas). Araras - SP. Brasil.

E-mail: pereiramb@gmail.com

http://orcid.org/0000-0003-3276-9407 


\section{Rosana Teresa Onocko Campos}

Docente da Faculdade de Ciências Médicas da Universidade Estadual de Campinas (Unicamp). Campinas - SP. Brasil.

E-mail: rosanaoc@mpc.com.br

(iD) http://orcid.org/0000-0003-0469-5447

Endereço para envio de correspondência:

Universidade Estadual de Campinas, Faculdade de Ciências Médicas da Unicamp, Departamento de Saúde Coletiva da FCM/Unicamp.

Rua Tessália Vieira de Camargo, 126, Barão Geraldo. CEP: 13084-971. Campinas - SP. Brasil.

Recebido 12/09/2017

Aceito 09/08/2018

Received 09/12/2017

Approved 08/09/2018

Recibido 12/09/2017

Aceptado 09/08/2018

Como citar: Bigatão, M. R., Pereira, M. B., \& Campos, R. T. O. (2019). Ressignificando um castelo: Um olhar sobre ações de saúde em rede. Psicologia: Ciência e Profissão, 39, 1-12. https://doi.org/10.1590/1982-3703003185242

How to cite: Bigatão, M. R., Pereira, M. B., \& Campos, R. T. O. (2019). Resignifying a castle: A look at health actions in network. Psicologia: Ciência e Profissão, 39, 1-12. https://doi.org/10.1590/1982-3703003185242

Cómo citar: Bigatão, M. R., Pereira, M. B., \& Campos, R. T. O. (2019). Resignificando un castillo: Una mirada sobre acciones de salud en red. Psicologia: Ciência e Profissão, 39, 1-12. https://doi.org/10.1590/1982-3703003185242 